\title{
MEIS2 (15q14) gene deletions in siblings with mild developmental phenotypes and bifid uvula: documentation of mosaicism in an unaffected parent
}

\author{
Bin Zhang ${ }^{1,2^{*}}$, Michel Liu $^{3}$, Chin-To Fong ${ }^{2}$ and M. Anwar lqbal ${ }^{1}$
}

\begin{abstract}
MEIS2 (Meis homeobox 2) encodes a homeobox protein in the three amino acid loop extension (TALE) family of highly conserved homeodomain-containing transcription regulators important for development. MEIS2 deletions/ mutations have been associated with cleft lip/palate, dysmorphic facial features, cardiac defects, as well as intellectual disability at a variable severity. Here we report on one familial case that two affected siblings carry the same non-mosaic $423 \mathrm{~kb}$ genomic deletion at 15q14 encompassing the entirety of CDIN1 and the last three exons (ex. 10, 11,12 ) of the MEIS2 gene, while their unaffected father is mosaic for the same deletion in about $10 \%$ lymphocytes. Both siblings presented with mild developmental delay and bifid uvula, while no congenital cardiac abnormalities were identified. The elder sister also showed syncopal episodes and mild speech delay and the father had atrial septal defects. This is the first report showing multiple family members inherit a genomic deletion resulting in a MEIS2 partial truncation from a mosaic parent. Taken all together, this study has important implications for genetic counseling regarding recurrence risk and also points to the importance of offering MEIS2 gene tests covering both point mutations and microdeletions to individuals with milder bifid uvula and developmental delay.
\end{abstract}

Keywords: MEIS2, Deletion, Mosaicism, Dosage effect, Orofacial clefts, Developmental delay, FISH, Chromosome microarray

\section{Introduction}

Orofacial clefts (OFCs) are among the most common human birth defects, affecting 1.56 per 1000 live births in North America [1], and present as a broad spectrum of phenotypes ranging from subclinical forms such as bifid uvula, to submucous cleft palate and velopharyngeal insufficiency, and to overt cleft palate. Sporadic OFCs are considered as a multifactorial polygenic trait caused by a number of genetic and environmental factors. Syndromic

\footnotetext{
*Correspondence: bin_zhang@urmc.rochester.edu

${ }^{1}$ Departments of Pathology and Laboratory Medicine, University

of Rochester Medical Center, 601 Elmwood Ave, Box 608, Rochester, NY 14642, USA

Full list of author information is available at the end of the article
}

OFCs are associated with developmental delay, dysmorphic features, or other major congenital anomalies, and mostly have a single genetic cause, either chromosomal or monogenic [2].

MEIS2 (Meis homeobox 2) is among the recentlyidentified OFC genes, and its disruption is considered to be the main causative factor contributing to the pathogenesis of chromosome 15q14 deletion syndrome [2-6]. MEIS2 encodes a homeobox protein in the three amino acid loop extension (TALE) family of highly conserved homeodomain-containing transcription regulators. It is expressed during early fetal brain, forelimb buds, developing hearts, and developing palatal shelves [7-9], and have been shown to be important for cranial and cardiac original author(s) and the source, provide a link to the Creative Commons licence, and indicate if changes were made. The images or other third party material in this article are included in the article's Creative Commons licence, unless indicated otherwise in a credit line to the material. If material is not included in the article's Creative Commons licence and your intended use is not permitted by statutory regulation or exceeds the permitted use, you will need to obtain permission directly from the copyright holder. To view a copy of this licence, visit http://creativecommons.org/licenses/by/4.0/. The Creative Commons Public Domain Dedication waiver (http://creativecommons.org/publicdomain/zero/1.0/) applies to the data made available in this article, unless otherwise stated in a credit line to the data. 
neural crest development $[10,11]$. MEIS2 protein may not be directly involved in complex DNA-binding, and most likely acts as PBX and HOX protein cofactors to form dimeric or trimeric complexes to enhance DNA binding specificity and affinity, and therefore regulate target gene expression [9]. It has been established that haploinsufficiency of the MEIS2 gene cause a syndromic form of OFCs, often presenting with cardiac defects, facial dysmorphism, as well as intellectual disability at a variable severity [2, 3, 6, 12-14]. MEIS2 mutations can vary as genomic microdeletions, single nucleotide variants (SNVs), or small insertions and deletions (indels), and most mutations occur de novo $[2,15]$. Rare diseasecausing mutation mosaicism has been reported [3]. Some MEIS2 missense mutations have been shown to cause more severe phenotypes than deletions, suggesting a possible dominant negative effect $[12,14]$. Multiple transcripts and alternative transcription start sites have been identified and studied for their potential regulatory function during development [16]. However, genotype and phenotype correlation studies between $3^{\prime}$ deletion and the entire gene deletion have not been conducted.

Here, we present one family with a $423 \mathrm{~kb}$ deletion encompassing the last three exons of the MEIS2 (15q14) gene in two siblings with bifid uvula and mild developmental delay and the same deletion as a mosaic lesion in the father with a congenital atrial septal defect. These findings could have implications for disease recurrence risk assessment and genetic counseling. We reviewed the literature and analyzed different mutation types, including loss-of-function microduplications/microdeletions and point mutations, along with the phenotypes associated. This study also points to the importance of offering MEIS2 gene tests covering both SNVs/indels and microdeletions to individuals with milder bifid uvula and developmental delay.

\section{Materials and methods Chromosome microarray (CMA)}

Chromosome array comparative genomic hybridization $(\mathrm{aCGH})$ experiments were performed using the SurePrint G3 Human CGH + SNP Microarray $4 \times 180 \mathrm{~K}$ (Agilent Technologies, CA), which contains approximately 110,712 oligonucleotides (60mers) for the detection of copy number variations (CNVs), along with 59,647 SNP probes for genotyping and detection of long stretches of contiguous homozygosity. A human genomic male/ female reference DNA sample, supplied by Agilent Technologies, is used as same sex controls for each analysis. DNA was extracted from the patient's peripheral blood using QIAamp ${ }^{\circledR}$ DNA Blood Mini Kit (Cat \# 51104, Qiagen Inc., Valencia, CA). Arrays were prepared in accordance with the manufacturer's instructions with an input amount of 500 ng of genomic DNA. Data were analyzed and visualized using the CytoGenomics v5.1.2 software (Agilent Technologies). The threshold for $\log 2$ ratios were -0.25 for losses and +0.25 for gains. The genomic linear positions are given relative to the GRCh37/hg19 genome assembly.

\section{Fluorescence in situ hybridization (FISH)}

Peripheral blood samples were cultured using standard cytogenetic methods for $72 \mathrm{~h}$ with phytohemagglutinin (PHA) stimulation. Fluorescence in situ hybridization was performed with standard techniques using the RP11450G24 bacterial artificial chromosome (BAC) probe (SpectrumGreen, Empire Genomics, NY) at 15q14 and the TelVysion probe for the subtelomeric region of chromosome 15: WI-5214(D15S936) (SpectrumOrange, Abbott Laboratories, Des Plaines, IL).

\section{Clinical report}

The proband (II-2 in Fig. 1a) was then a 3-year-old female and was referred to the University of Rochester Medical Center (URMC) genetics clinic for evaluation of developmental delay and bifid uvula. Her prenatal history is unremarkable except for intrauterine growth restriction (IUGR). She was born with a full-term gestation. Her birth weight is $2.69 \mathrm{~kg}$ (8.85 percentile, Z-score - 1.35) and her birth length is $48.26 \mathrm{~cm}(33.51$ percentile, Z-score -0.43$)$. She never crawled, scooted around at one year of age, or walked at 18 months. She had mild speech delay and talked clearly with words around 14-15 months. Now she speaks in full sentences. Her growth is adequate despite initial IUGR and she has global developmental delay.

She has a history of recurrent ear infections and had tympanostomy tubes placed. She started to have intermittent syncopal episodes lasting 3-5 $\mathrm{s}$ at 1.5 years of age. Her electrocardiogram (ECG) is normal with a normal QTc. An echocardiogram confirms normal cardiac anatomy and function with an intact atrial septum. She has no cardiac abnormalities and her previous prominent foramen ovale flap noted in utero was interpreted as a normal variant. The syncopal episodes of a short duration are not considered to represent a primary cardiac event.

The brother (II-3 in Fig. 1a) was 12 months old at the time of referral and presents with mild developmental delay and bifid uvula. His prenatal history is unremarkable except for oligohydramnios. He was born with a gestational age of $36 \mathrm{w} 5 \mathrm{~d}$. His birth weight is $2.58 \mathrm{~kg}$. (5.69 percentile, Z-score -1.58 ) and his birth length is $48.26 \mathrm{~cm}$ (22.88 percentile, Z-score-0.74). He did not start walking until he was about 21 months old. He is the 5 th percentile for weight. He has slight genu valgum bilaterally. There is no other congenital malformation. He 


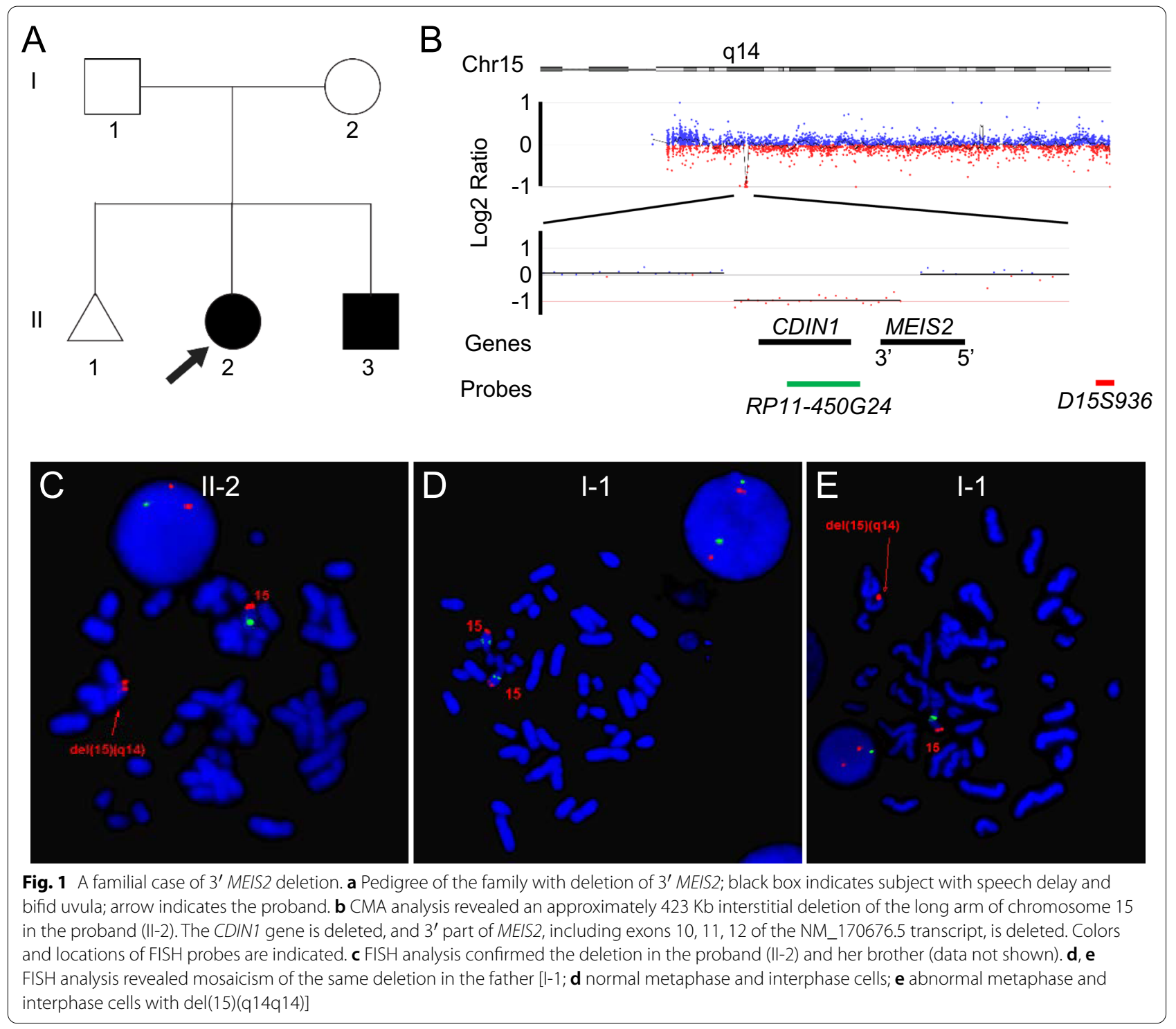

is small but growing steadily along his own growth curve. There is no history of cyanosis, shortness of breath, exercise intolerance, fatigue, tachycardia, diaphoresis, syncope, or presyncope. There is no history of seizures.

The father (I-1 in Fig. 1a) had an atrial septal defect per report. There are no family history of developmental delay and bifid uvula.

\section{Results}

Chromosome microarray analysis revealed a non-mosaic $\sim 423 \mathrm{~kb}$ genomic deletion at $15 \mathrm{q} 14$ (chr15:36808211-37231497) encompassing the entirety of CDIN1 and $3^{\prime}$ end of the MEIS2 gene (NM_170676.5) in the proband (II-2) and her younger brother (Fig. 1a, b). The breakpoint defined between chr15:37231467 (the first deleted $3^{\prime}$ probe) and chr15:37285154 (the first undeleted $3^{\prime}$ probe) is within intron 9 of the MEIS2 gene and causes deletion of last three exons (ex. 10, 11, 12) of the NM_170676.5 transcript. FISH analysis with a BAC probe (RP11-450G24, chr15:36960019-37122831) confirmed the deletion (Fig. 1c). The father is mosaic for the same deletion in $12 \%(3 / 25)$ metaphase and 9\% (18/200) interphase cells of his peripheral blood (Fig. 1d, e).

\section{Discussion}

MEIS2 mutations, including genomic microdeletions and SNVs and indels, have been recently identified to cause syndromic phenotypes, including palatal defects, congenital cardiac defects, and intellectual disability at variable severity and penetrance $[2,6,14]$. We report a genomic 
microdeletion affecting the last three exons (ex. 10, 11, and 12) of the MEIS2 gene (NM_170676.5) and CDIN1 in two siblings, presenting mild developmental delay and bifid uvula. The father is mosaic for the same deletion, but did not present with the syndromic phenotypes. The CDIN1 gene encodes a novel restriction endonuclease, belonging to the Holliday junction resolvase family, and two missense mutations have been identified to be responsible for autosomal recessive congenital dyserythropoietic anemia type Ib (OMIM \#615631) [17]. There are no evidence that heterozygous missense mutation or deletion of the CDIN1 gene causes disease in the literature. The pLI score, the probability of intolerance to a Loss of Function (LoF) mutation, is 0 for the CDIN1 gene per the gnomAD database, indicating that heterozygous deletion is likely tolerated and not involved in the phenotype of the siblings of this study. Haploinsufficiency of MEIS2 with the pLI score of 1 is considered to be responsible for a syndromic phenotype with cleft palate, intellectual disability, heart defects, and dysmorphic features [2]. Up to date, 20 unrelated patients with MEIS2 mutations and 29 unrelated patients with deletions or exonic duplications have been identified by sequencing analysis and array-CGH analysis [2, 14, 15, 18-21]. Interestingly, a recent genotype and phenotype correlation study showed that MEIS2 missense variants are associated with a phenotype that overlaps but is broader than that reported in individuals with gross deletions of MEIS2 [14], suggesting a dominant negative effect. In contrast, a few individuals with genomic microdeletions present a very mild phenotype [22].

MEIS2 is an evolutionary-conserved homeobox gene, encoding a homeodomain (HD)-containing transcriptional activator regulating cell proliferation and differentiation of various tissues and organs during development [23, 24]. Multiple transcript isoforms of MEIS2, of particular, the $3^{\prime}$ splice variants involving exons 11 and 12, have been identified to form four major protein isoforms with different $C$ termini in human [16], which can assemble largely non-overlapping interactomes, allowing them to recruit different proteins to the regulatory regions of target genes [25]. Interestingly, a truncated HD-less isoform of $h t h$, the MEIS2 homolog in drosophila, is expressed alongside the canonical, full-length isoform, can function without directly binding DNA [26], implicating that human MEIS2 may function similarly when the HD domain is completely or partially truncated. $3^{\prime}$ MEIS2 truncating microdeletions could cause different phenotypic severity depending on the number of $3^{\prime}$ MEIS2 exons deleted.

The last three exons (ex. 10-12) of MEIS2 were deleted in two siblings reported in this study (Table 1). Two other cases in the literature were identified to have deletions encompassing the last three or fewer exons (Patient A and Patient $\mathrm{H}$ in [2], Table 1). Interestingly, all four cases did not show congenital cardiac defects, which occur in $72 \%(13 / 18)$ of individuals with MEIS2 mutations (SNVs or indels), and 38\% (10/26) of other microdeletion and duplication cases. The two siblings of this study only present mild developmental delay and bifid uvula, while patient $\mathrm{A}$ and patient $\mathrm{H}$ didn't show palate defects, which occur in $80 \%(16 / 20)$ of MEIS2 mutations, and $85 \%(22 / 26)$ of other microdeletion and duplication cases (Table 1). The average walking age for these four cases are $24 \mathrm{mo}$, which is not significantly different from $26 \mathrm{mo}$ for cases with MEIS2 deletion and a range of $14 \mathrm{mo}-4 \mathrm{y}$ for cases with microdeletion and duplications (Table 1). Only one of four cases presented with dysmorphic facial features, compared to $100 \%$ (17/17) of MEIS2 mutations, and $83 \%(10 / 12)$ of other microdeletion and duplication cases (Table 1). In addition, patient $A$ and patient $H$ have intellectual disability (ID), while two siblings of this study were not evaluated for ID due to their age. It needs to be pointed out that patient $\mathrm{H}$ has a $2.92 \mathrm{Mb}$ deletion (chr15:34308789-37231638) encompassing a number of protein coding genes which likely contribute to phenotypes of facial dysmorphism and ID (Table 1).

MEIS2 mosaicism is rare and has not been frequently documented in the literature. A mosaic $123 \mathrm{~kb}$ deletion encompassing only the exon 9 of MEIS2 was reported to be responsible for a cleft soft palate, ventricular septal defect, and bilateral moderate hearing loss [3]. Su et al, recently reported a boy with the phenotypic spectrum, including atrial and ventricular septal defects, developmental delay, facial dysmorphism, primary neutropenia, branchial anomalies, and complex genital anomalies, inherits a MEIS2 p.R333del mutation from his unaffected father with a low-level mosaicism [19]. Our study represents the first report showing that multiple family members inherit a genomic deletion resulting in a $3^{\prime}$ MEIS2 partial truncation from a mosaic father, who is healthy other than an atrial septal defect (ASD). However, the ASD in the father is likely not related to the mosaic $3^{\prime}$ MEIS2 deletion as mosaicism level can vary significantly among tissues and it was determined only in peripheral blood in this study. In addition, the molecular origin of the mosaicism, as a de novo event arising post-zygotically or a gene conversion following a germline mutation, remains to be defined. These findings would have implications for recurrence risk counseling for families.

Interestingly, a $3^{\prime}$ MEIS2 deletion only affecting last three exons ([hg19] chr15:37163900_37195301, NSV569198 in the Database of Genomic Variants (DGV)) has been reported in a normal individual, that served as a control used for a study to establish a copy number variation morbidity map of developmental 
Table 1 Clinical features associated with different MEIS2 variants

\begin{tabular}{|c|c|c|c|c|c|c|}
\hline & MEIS2 dels/dups ${ }^{\#}$ & $\begin{array}{l}\text { MEIS2 } \\
\text { mutations }\end{array}$ & Patient $A^{\#}$ & Patient $\mathrm{H}^{\#}$ & II-2; II-3 & $\mathrm{I}-1$ \\
\hline \multirow[t]{2}{*}{$\begin{array}{l}\text { Mutation size/ } \\
\text { coordinates }^{\$}\end{array}$} & Variable & SNVs and indels & $\begin{array}{l}194 \mathrm{~Kb} / \\
\text { chr15:36989551_37184183 }\end{array}$ & $\begin{array}{l}2.92 \mathrm{Mb} / \\
\text { chr15:34308789_37231638 }\end{array}$ & \multicolumn{2}{|c|}{$\begin{array}{l}423 \mathrm{~Kb} / \mathrm{chr15} \text { : } \\
\text { chr15:36808211-37231497 }\end{array}$} \\
\hline & & & & & Non-mosaic & Mosaic \\
\hline Mutation types & $\begin{array}{l}\text { Disruption includ- } \\
\text { ing exon } 9^{*}\end{array}$ & $\begin{array}{l}7 \text { nonsense; } 6 \\
\text { missense; } 3 \text { splic- } \\
\text { ing; } 2 \text { in-frame } \\
\text { del; } 2 \text { frameshift } \\
\text { mutation }\end{array}$ & Exon 12 deletion* & Exon 10-12 deletion* & \multicolumn{2}{|c|}{ Exon 10-12 deletion* } \\
\hline Inheritance Mode & $\begin{array}{l}19 \text { de novo; } 2 \\
\text { familial;8 NA }\end{array}$ & 18 de novo/2 pat & de novo & de novo & pat & de novo \\
\hline Palate defects & 22/26; $1 \mathrm{NA}$ & $16 / 20$ & - & - & Bifid uvula & - \\
\hline Cardiac defects & 10/26; $1 \mathrm{NA}$ & 13/18; 2 NA & - & - & - & ASD \\
\hline $\begin{array}{l}\text { Developmental } \\
\text { delay }\end{array}$ & + & + & + & + & Mild & - \\
\hline Walked@ & $14 \mathrm{mo}-4 \mathrm{y}$ & $\begin{array}{l}\text { 18mo (Giliberti } \\
\text { et al. 2020); } 26 \mathrm{mo} \\
\text { (11 cases reviewed } \\
\text { in Verheije et al., } \\
\text { 2019); 30mo } \\
\text { (Santoro et al. } \\
\text { 2021); 32mo (Su } \\
\text { et al. 2020); NA (6 } \\
\text { cases from Sriv- } \\
\text { astava et al. 2018, } \\
\text { Hildebrand et al. } \\
\text { 2020, Douglas } \\
\text { et al. 2018) }\end{array}$ & $27 \mathrm{mo}$ & 30mo & $\begin{array}{l}\text { II-2: 18mo; II-3: } \\
21 \mathrm{mo}\end{array}$ & - \\
\hline Facial features & $\begin{array}{l}\text { Variable: 10/12 (14 } \\
\text { cases from Ver- } \\
\text { heije et al. 2019) }\end{array}$ & $\begin{array}{l}\text { Variable; 17/17; } \\
3 \text { NA }\end{array}$ & - & $\begin{array}{l}\text { Anteverted nares, asym- } \\
\text { metric ears with abnormal } \\
\text { helix }\end{array}$ & - & - \\
\hline $\begin{array}{l}\text { Intellectual dis- } \\
\text { ability }\end{array}$ & $19 / 21 ; 8 \mathrm{NA}$ & $20 / 20$ & + & + & NA & - \\
\hline
\end{tabular}

NA not available, + phenotype observed, - phenotype not observed, ASD atrial septal defect, SNV single nucleotide variant, dels/dups microdeletions/ microduplications, indels small insertion/deletions, pat paternal, mo months, $y$ years

\# Reference [2]—Verheije et al. (2019)

\& Reference [2]—Verheije et al. (2019) [14],_Douglas et al. (2018), [15]—Giliberti et al. (2020), [18]—Hildebrand et al. (2020), [19]—Su et al. (2020), [20]—Fujita et al. (2016), [21] —Santoro et al. (2021), [22] — Srivastava et al. (2018)

\$ Genomic coordinates are based on the genome assemble GRCh37/hg19

*Exon definition is based on the NM_170676.5 transcript

delay [27]. This suggests that phenotype in such individual could be very subtle, if any. This finding points to the importance of offering MEIS2 gene tests covering both SNVs/indels and microdeletions to individuals with milder bifid uvula and developmental delay. This report also raises the possibility that cryptic or tissuespecific mosaicism of genetic lesions involving the MEIS2 gene could cause diseases and may pose a diagnostic challenge.

Taken together, it is possible that some $3^{\prime}$ MEIS2 deletions could produce a truncated partial functional protein and therefore act as a hypomorphic mutation, instead of nullimorphic (complete loss of function), causing a mild phenotype. Further studies that provide experimental evidence, such as RNA or protein analysis, are necessary to confirm partial expression of $5^{\prime}$ MEIS2 and this hypothesis. In vivo functional studies are warranted to define three types of MEIS2 mutations-dominant negative alleles (missense variants) [14], loss-of-function alleles (whole gene deletion or exonic deletion/duplication involving exon 9 and upstream) [2], and hypothesized hypomorphic alleles (deletions involving exon 10 and downstream) [2, 22, 27]. More clinical cases are needed to establish genotype-phenotype correlation. 


\section{Abbreviations}

MEIS2: Meis homeobox 2; TALE: Three amino acid loop extension; OFC: Orofacial clefts; CMA: Chromosome microarray; aCGH: Array comparative genomic hybridization; CNVs: Copy number variants; SNP: Single nucleotide polymorphism; FISH: Fluorescence in situ hybridization; PHA: Phytohemagglutinin; BAC: Bacterial artificial chromosome; URMC: University of Rochester Medical Center; IUGR: Intrauterine growth restriction; ECG: Electrocardiogram; SNVs: Single nucleotide variants; Indels: Insertions and deletions; HD: Homeodomain; OMIM: Online Mendelian Inheritance in Man; ID: Intellectual disability; ASD: Atrial septal defect; DGV: Database of Genomic Variants.

\section{Acknowledgements}

We thank the family and patients for their participation in this study.

\section{Authors' contributions}

$B Z$, CF, and MAI conceived, designed, and performed the experiments, and wrote the paper. $B Z$ and $M L$ revised the manuscript. All authors read and approved the final manuscript.

\section{Funding}

This work was supported by the Department of Pathology and Laboratory Medicine at University of Rochester Medical Center. No other funding was received.

\section{Availability of data and materials}

The raw data of $\mathrm{CGH}$ and FISH were available upon request.

\section{Declarations}

\section{Ethical approval and consent for participate}

This study (STUDY 00000060) was approved by Institutional Review Board of University of Rochester Medical Center. All information in this report has been de-identified. Consent to participate and for publication is not required for this single case study not including identifiable information or images.

\section{Competing interests}

The authors have declared that no competing interests.

\section{Author details}

${ }^{1}$ Departments of Pathology and Laboratory Medicine, University of Rochester Medical Center, 601 Elmwood Ave, Box 608, Rochester, NY 14642, USA. ${ }^{2}$ Department of Pediatrics, University of Rochester Medical Center, 601 Elmwood Ave, Rochester, NY 14642, USA. ${ }^{3}$ Renal-Electrolyte and Hypertension Division, Perelman School of Medicine, University of Pennsylvania, Philadelphia, PA 19104, USA.

Received: 1 May 2021 Accepted: 12 October 2021

Published online: 20 December 2021

\section{References}

1. Panamonta $\vee$, et al. Global birth prevalence of orofacial clefts: a systematic review. J Med Assoc Thai. 2015;98(Suppl 7):S11-21.

2. Verheije $R$, et al. Heterozygous loss-of-function variants of MEIS2 cause a triad of palatal defects, congenital heart defects, and intellectual disability. Eur J Hum Genet. 2019:27(2):278-90.

3. Crowley MA, et al. Further evidence for the possible role of MEIS2 in the development of cleft palate and cardiac septum. Am J Med Genet A. 2010;152A(5):1326-7.

4. Erdogan F, et al. Characterization of a $5.3 \mathrm{Mb}$ deletion in $15 \mathrm{q} 14$ by comparative genomic hybridization using a whole genome "tiling path" BAC array in a girl with heart defect, cleft palate, and developmental delay. Am J Med Genet A. 2007:143A(2):172-8.

5. Chen CP, et al. A 5.6-Mb deletion in $15 q 14$ in a boy with speech and language disorder, cleft palate, epilepsy, a ventricular septal defect, mental retardation and developmental delay. Eur J Med Genet. 2008;51(4):368-72.

6. Johansson S, et al. Haploinsufficiency of MEIS2 is associated with orofacial clefting and learning disability. Am J Med Genet A. 2014;164A(7):1622-6.

7. Cecconi F, et al. Expression of Meis2, a Knotted-related murine homeobox gene, indicates a role in the differentiation of the forebrain and the somitic mesoderm. Dev Dyn. 1997;210(2):184-90.

8. Yakushiji-Kaminatsui N, et al. RING1 proteins contribute to early proximaldistal specification of the forelimb bud by restricting Meis2 expression. Development. 2016;143(2):276-85.

9. Wang $L$, et al. The transcriptional regulator MEIS2 sets up the ground state for palatal osteogenesis in mice. J Biol Chem. 2020;295(16):5449-60.

10. Machon $\mathrm{O}$, et al. Meis 2 is essential for cranial and cardiac neural crest development. BMC Dev Biol. 2015;15:40.

11. Fabik J, et al. Neural crest cells require Meis 2 for patterning the mandibular arch via the Sonic hedgehog pathway. Biol Open. 2020;9(6):66

12. Louw JJ, et al. MEIS2 involvement in cardiac development, cleft palate, and intellectual disability. Am J Med Genet A. 2015;167A(5):1142-6.

13. Shimojima $K$, et al. A 15 q14 microdeletion involving MEIS2 identified in a patient with autism spectrum disorder. Hum Genome Var. 2017:4:17029.

14. Douglas $\mathrm{G}$, et al. De novo missense variants in MEIS2 recapitulate the microdeletion phenotype of cardiac and palate abnormalities, developmental delay, intellectual disability and dysmorphic features. Am J Med Genet A. 2018;176(9):1845-51.

15. Giliberti A, et al. MEIS2 gene is responsible for intellectual disability, cardiac defects and a distinct facial phenotype. Eur J Med Genet. 2020;63(1):103627.

16. Schulte D, Geerts D. MEIS transcription factors in development and disease. Development. 2019;146(16):652.

17. Babbs C, et al. Homozygous mutations in a predicted endonuclease are a novel cause of congenital dyserythropoietic anemia type I. Haematologica. 2013;98(9):1383-7.

18. Hildebrand MS, et al. Severe childhood speech disorder: gene discovery highlights transcriptional dysregulation. Neurology. 2020;94(20):e2148-67.

19. Su JX, et al. MEIS2 sequence variant in a child with intellectual disability and cardiac defects: expansion of the phenotypic spectrum and documentation of low-level mosaicism in an unaffected parent. Am J Med Genet A. 2020;6:66

20. Fujita A, et al. De novo MEIS2 mutation causes syndromic developmental delay with persistent gastro-esophageal reflux. J Hum Genet. 2016:61(9):835-8

21. Santoro C, et al. A novel MEIS2 mutation explains the complex phenotype in a boy with a typical NF1 microdeletion syndrome. Eur J Med Genet. 2021;64(5):104-90.

22. Conte F, et al. Systematic analysis of copy number variants of a large cohort of orofacial cleft patients identifies candidate genes for orofacial clefts. Hum Genet. 2016;135(1):41-59.

23. Huang $\mathrm{H}$, et al. MEIS $\mathrm{C}$ termini harbor transcriptional activation domains that respond to cell signaling. J Biol Chem. 2005;280(11):10119-27.

24. Agoston Z, et al. Genetic and physical interaction of Meis2, Pax3 and Pax7 during dorsal midbrain development. BMC Dev Biol. 2012;12:10.

25. Gross A, et al. Tumorigenic and antiproliferative properties of the TALEtranscription factors MEIS2D and MEIS2A in neuroblastoma. Cancer Res. 2018;78(8):1935-47.

26. Noro B, et al. Distinct functions of homeodomain-containing and homeodomain-less isoforms encoded by homothorax. Genes Dev. 2006:20(12):1636-50.

27. Cooper GM, et al. A copy number variation morbidity map of developmental delay. Nat Genet. 2011:43(9):838-46.

\section{Publisher's Note}

Springer Nature remains neutral with regard to jurisdictional claims in published maps and institutional affiliations. 